\title{
Synergy of IoT and AI in Modern Society: The Robotics and Automation Case
}

\author{
Spyros G Tzafestas* \\ National Technical University of Athens, Greece
}

Submission: August 15, 2018; Published: September 12, 2018

*Corresponding author: Spyros G Tzafestas, National Technical University of Athens, Greece; Tel: +30-210-6524000; Email: tzafesta@cs.ntua.gr

Abstract

The Internet of Things (IoT) is a recent revolution of the Internet which is increasingly adopted with great success in business, industry, healthcare, economic, and other sectors of modern information society. In particular, IoT supported by artificial intelligence enhances considerably the success in a large repertory of every-day applications with dominant one's enterprise, transportation, robotics, industrial, and automation systems applications. Our aim in this article is to provide a global discussion of the main issues concerning the synergy of IoT and AI, including currently running and potential applications of great value for the society. Starting with an overview of the IoT and AI fields, the article describes what is meant by the concept of 'IoT-AI synergy', illustrates the factors that drive the development of 'IoT enabled by AI', and summarizes the concepts of 'Industrial IoT' (IIoT), 'Internet of Robotic Things' (IoRT), and 'Industrial Automation IoT (IAIoT). Then, a number of case studies are outlined, and, finally, some IoT/AI-aided robotics and industrial automation applications are presented.

Keywords: Artificial intelligence (AI); Internet of things (IoT); Machine learning (ML); Cognitive IoT; Internet of robotic things (IoRT); IoT- aided robotics; Industrial IoT (IIoT); IoT- aided industrial automation; IoT-aided manufacturing

\section{Introduction}

As the IBM Institute for Business Value has pointed out, the full potential of the Internet of Things (IoT) can only be realized with the introduction of Artificial Intelligence (AI). Actually, IoT and AI are umbrella terms. The IoT can be described as things/objects in our environment being connected to provide seamless communication and contextual services. IoT involves a tremendous number of connections of things to things and things to humans, and therefore it is more complex and dynamic than the Internet. According to IDC's Worldwide IoT taxonomy (2015), the IoT market place is estimated to be worth 1.7 trillion US Dollars, with the biggest portion (35\%) being hardware followed by services (27\%), connectivity (22\%), and software $(16 \%)$.

As originally described by Minsky and McCarthy (the fathers of $\mathrm{AI}$ ), $\mathrm{AI}$ is any task carried out by a program or machine that, if a human performed the same task, one would say that human had to apply intelligence to accomplish the task. Today AI has a ubiquitous use in a large variety of applications of modern information society. Scientifically, AI is distinguished in:

A. Narrow AI that involves all intelligent systems that can carry-out specific tasks without being explicitly programmed how to do so, and

B. General AI which is a flexible form of intelligence that can learn how to perform a variety of different tasks.

Looking at IoT and AI one can easily see what both have in common, viz. data enhanced to information, to knowledge, to intelligence, and finally to decisions for specific purposes across a variety of everyday, enterprise, and industry/automation situations. With the AI synergy, IoT becomes smarter. Today the number of companies that embed AI (e.g., machine learning, intelligent reasoning) into their IoT endeavors is rapidly increasing. These companies see their capabilities to grow and their operational efficiency to improve, including a big reduction of unplanned down time. This indicates that companies that develop an IoT strategy, or evaluate a potential new IoT-based activity, or seek to obtain more value from an existing IoT application will get many benefits from the incorporation of AI methods and tools in their IoT endeavors.

The purpose of this article is to provide a global conceptual overview of the synergy of AI and IoT with emphasis on its application in robotics and automation. Specifically, the article:

a) Discusses the ontological questions 'what is IoT' and 'what is AI'.

b) Presents fundamental issues about the 'synergy of IoT and $\mathrm{AI}^{\prime}$ or 'IoT enabled by AI'.

c) Outlines the concepts of 'Industrial Internet of Things' (IIoT), 'Internet of Robotic Things' (IoRT), and 'Industrial Automation Internet of Things' (IAIoT).

d) Outlines a number of case studies (home automation oil-field production, smart robotics, smart manufacturing, and smart factory). 


\section{Robotics \& Automation Engineering Journal}

e) Summarizes the field of IoT-aided robotics.

f) Discusses an application of IoT-aided industrial automation.

\section{What is IOT?}

The term "Internet of Things" (IoT) is now widely used, but so far there is not a unique common definition or understanding of what this term encompasses. The term Internet of things was first used in 1999 by Kevin Ashton, director of AutoID Center (MIT), working on networked "radio-frequency identification" (RFID) infrastructures [1-5]. He coined this term in order to reflect his envisioning of a world in which all electronic devices are networked and every object (physical or electronic) is tagged with information pertinent to that object [2]. The Internet of things, which is sometimes referred to as Internet of Objects (IoO), is actually a new enhancement of the Internet, and the things/objects make them recognizable by communicating information about them. They can get information about them accumulated by other objects and things, or they can be elements of high-level services.

From the many alternative definitions of the term IoT, we select here the definition given by the IEEE (IoT Initiative, 2015) which is divided in two parts [6]:

a. Part 1: Definition for a small environment scenario: "An IoT is a network that connects uniquely identifiable 'Things' to the Internet. The 'Things' have sensing/actuator and potential programmability capabilities. Through the exploitation of unique identification and sensing, information about the 'Thing' can be collected and the state of the 'Thing' can be changed from anywhere anytime, by anything" [6].

b. Part 2: Definition for a large environment scenario (where a large number of "Things" can be interconnected to provide complex services and enable the execution of complex processes): "Internet of Things envisions a selfconfiguring, adaptive, complex network that interconnects 'things' to the Internet through the use of standard communication protocols. The interconnected things have physical or virtual representation in the digital world, sensing/actuator capability, a programmability feature, and are uniquely identified. The representation contains information including the thing's identity, status, location or any other business, social or privately relevant information. The things offer services, with or without human intervention, through the exploitation of a unique identification, data capture and communication, and actuation capability. The service is exploited through the use of intelligent interfaces and is made available anywhere, anytime, and for everything taking security in consideration" [6].

Actually, IoT is distinguished in three interaction categories [1]:
I. People to people IoT.

II. People to things (objects, machines) IoT.

III. Things/machines to things/machines IoT.

'Things' refer in general to everyday objects that are readable, recognizable, locatable, and addressable via information sensing devices, and/or controllable via the Internet, irrespectively of the communication means employed (RFID, wireless LAN, WAN, etc.). IoT is interdisciplinary, and according to Atzori et al. [2], falls in the following three paradigms:

i. Internet-oriented (middleware).

ii. Things-oriented (sensors).

iii. Semantic-oriented (knowledge).

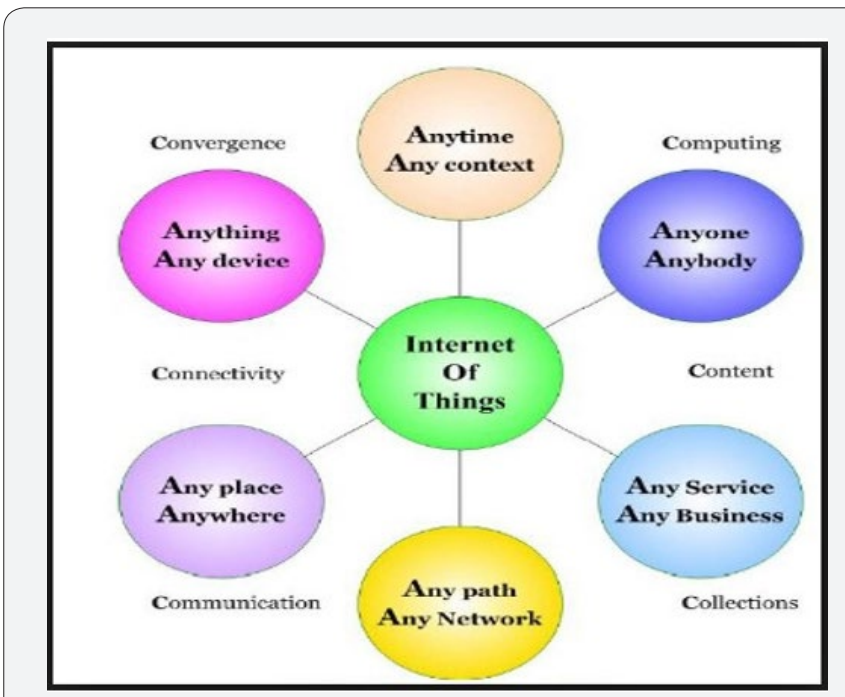

Source: [1].

Figure 1: Interconnections in loT.

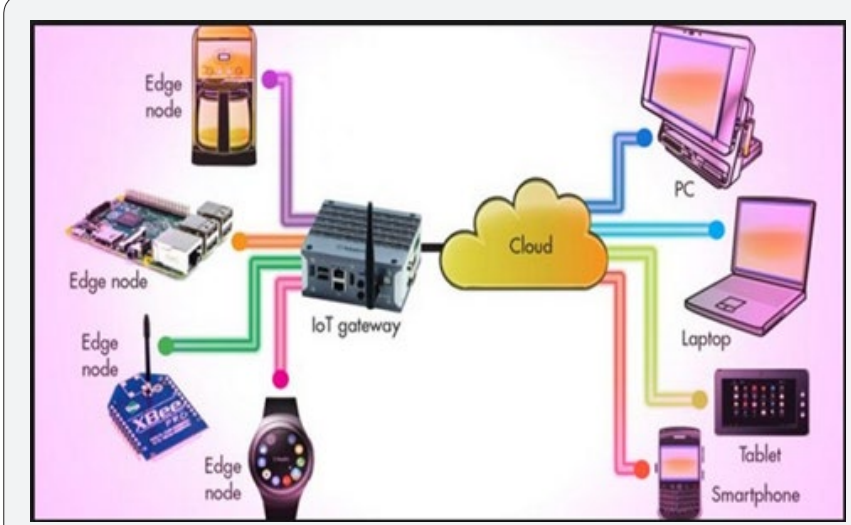

Source: https://www.electronicdesign.com/iot/iot-frameworksdevelopment-dominate-software-trends-2016

Figure 2: Interconnections in loT.

It is remarked that IoT is particularly important and useful in application domains that belong to all the above paradigms. Actually, IoT is a new development of the Internet which aims at enabling 'Things' to be connected anytime (any context) at 
anyplace (anywhere) with anything (any device) and anyone (anybody) using any path or network and any service or business (Figure1) [1].

Figure 2 gives a schematic representation of IoT connectedness through gateway and cloud.

Because of its characteristics, IoT is very rapidly penetrating almost all areas of our lives. The fundamental characteristics of IoT are $[1,7]$ :

a. Connectivity: Connectivity makes possible network accessibility and compatibility. Anything can be interconnected with the overall IoT communication and information facilities. Compatibility means that 'things' have the common ability to generate and consume data.

b. Heterogeneity: IoT components and devices are heterogeneous since they are based on different platforms and networks. They can communicate and interact with other devices and service platforms via a variety of networks.

c. Tremendous scale: The IoT number of things and devices that communicate and interact with each other, and have to be managed, is at least one order of magnitude bigger than that of the present internet.

d. Dynamic changes: The state and number of components and devices of IoT change dynamically (e.g., alternating connection and disconnection, changing position and speed, etc.).

e. Safety: IoT should be designed for safety of personal data, physical safety, and human well-being. Securing the end points, the networks, and the data travelling through them, implies that we create a security paradigm that we scale.

f. Small devices: Devices are becoming smaller and smaller, cheaper, and more powerful over time.

2) IoT uses small devices built for several tasks and purposes, to achieve its accuracy, scalability and versatility.

a. Autonomous agency: IoT gives an environment for getting augmented human agency, sometimes reaching the point of spontaneous unexpected interventions that are not directly caused by human beings.

b. Pervasiveness /ubiquity: IoT embeds computational capability into everyday objects and makes them effectively communicate and perform desired tasks in a way that minimizes the human need to interact with computers as computers. IoT devices are network-connected devices and always available. IoT makes computing truly ubiquitous and opens new horizons for the society, the economy, and the individual.

c. Ontological vagueness: Human beings, physical objects, and artifacts may not be clearly distinguished due to the deliberate transformation of entities of one type into entities of another type via tagging, engineering, and absorption into a network of artifacts. Criteria to deal with ambiguous identity and system boundary should be developed and used.

d. Distributed control: In IoT the control is not centrally exerted but, because of the enormous number of nodes, it has a distributed form and exhibits emergent features and behaviors which require proper distributed control.

e. Expressing: This feature enables interactivity with people and the physical world. In all cases, 'expressing' helps us to create products/things that interact intelligently with the real world and the environment.

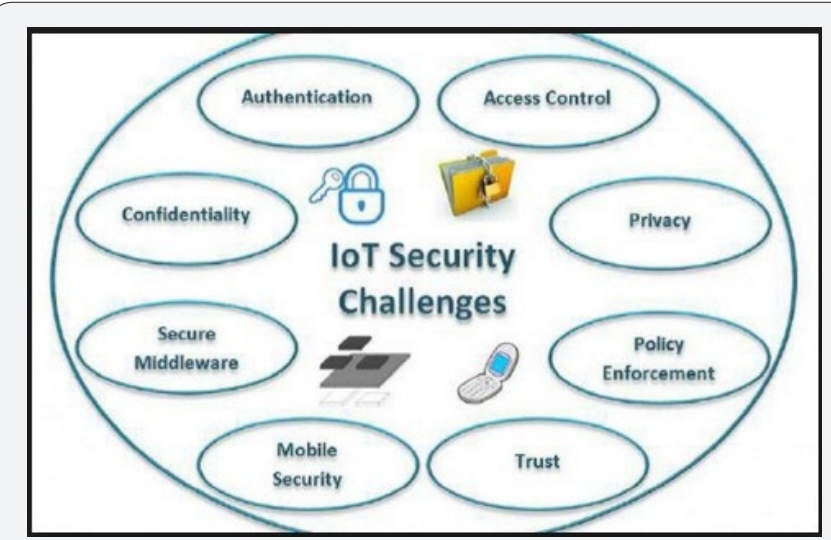

Source: https://www.semanticscholar.org/paper/Security-Issuesin-Internet-of-Things-(-loT-)-\%3A-A-Balte-Kashid/264ee1f311a9 bd5762c8d4de6cd5e54647084413

Figure 3: Primary challenges of loT security.

The big challenge of IoT is the security issue that involves the protection of access to equipment (e.g., internet connected home or connected car, etc.) and the protection of customer and company data. It is noted that with customer and company data, a different kind of security is needed. Other security challenges of IoT are depicted in Figure 3. An aspect of IoT that should not be ignored is the fact that IoT devices, data, and networks need to be monitored in real time, otherwise we may not have success with IoT.

\section{What is AI?}

The artificial intelligence (AI) field is concerned with intelligent machines, or rather with embedding intelligence to computers, i.e., "artificial intelligence is the science and engineering of making intelligent machines" [8]. Today, AI has become an important element of computer industry helping to solve extremely difficult problems of society. AI includes the expert systems which are computer programs that simulate the reasoning and performance of human experts. Alternatively, one can say that an ES is a computer application which solves complex problems that would otherwise require extensive human expertise. To this end, it simulates the human reasoning problem by using specific rules or objects representing the human expertise. 
Some of the problems that fall in the framework of AI are [8-14]:

a. Game playing.

b. Theorem proving.

c. General problem solving.

d. Natural language understanding.

e. Machine learning.

f. Pattern recognition.

g. Perception and cognition. h. Symbolic mathematics.

i. Medical diagnostics.

j. Fault diagnosis/restoration of technological systems.

k. AI- based/Expert control.

A map that shows 'what is AI' is given in Figure 4. It builds on mathematics, philosophy, cognitive psychology, and biology. Its methods are distinguished in knowledge-based methods, behavioral methods, and subsymbolic methods, and has both a scientific and a technological content.

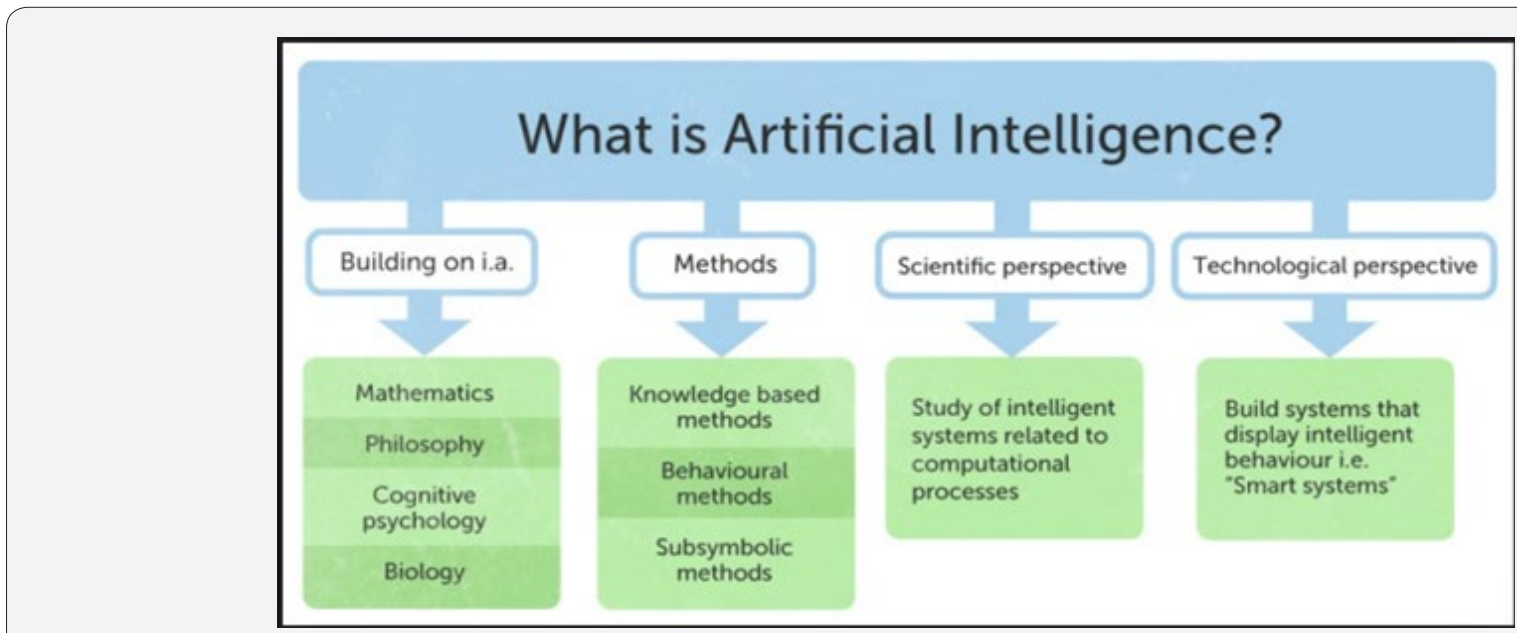

Source: https://www.semanticscholar.org/paper/Security-Issues-in-Internet-of-Things-(-loT-)-\%3A-A-Balte-Kashid/264ee1f311a9bd5762c8 d4de6cd5e54647084413

Figure 4: Primary challenges of loT security.

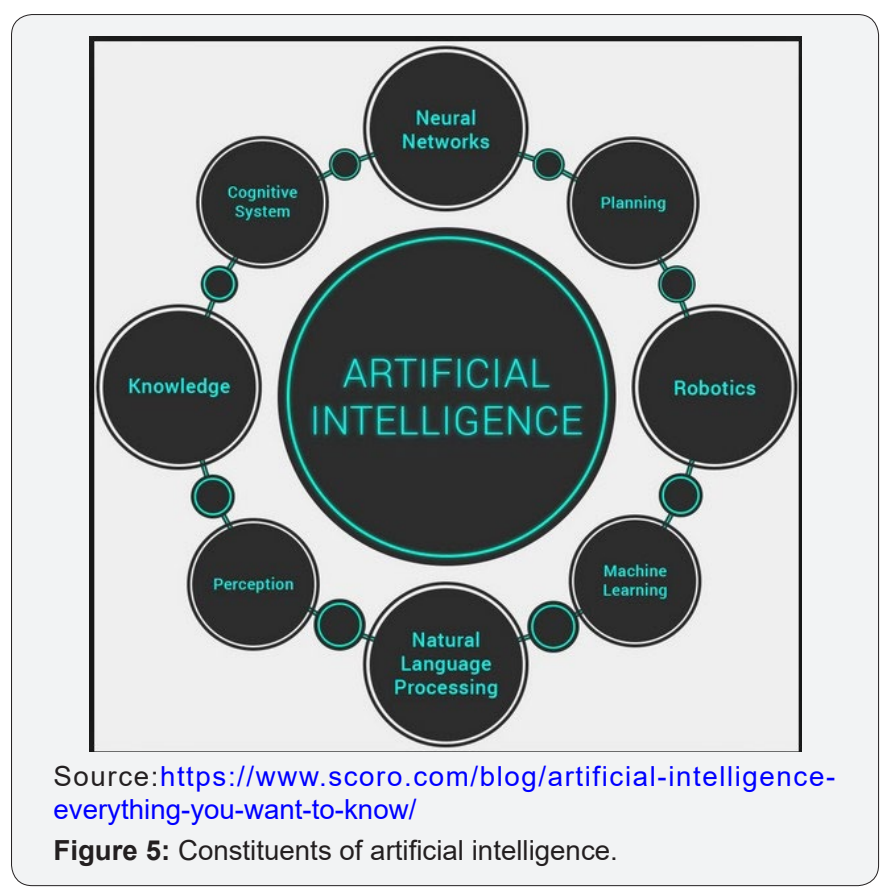

Figure 5 shows the constituents of AI (of course nonexhaustively). The robotics part that really belongs to AI includes all intelligent algorithms that perform path/task planning, local/global navigation, and intelligent/knowledgebased control.
The AI process that is mostly used in IoT is machine learning. It is difficult to define machine learning uniquely, since it ranges from the addition of any single fact or a new piece of new knowledge to a complex control strategy, or a proper rearrangement of system structure, and so on. A useful class of machine learning is the automated learning which is the process (capability) of an intelligent system to enhance its performance through learning, i.e., by using its previous experience. In other words, intelligent machines can learn to operate and improve by observing, classifying, and correcting their errors just like humans do. Five basic automated learning paradigms are:

A. Concept learning.

B. Inductive learning (learning by examples).

C. Learning by discovery.

D. Connectionist/neural network learning.

E. Learning by analogy.

Below we list three working machine learning systems:

a. IBM Watson: A question answering software system that can answer questions using machine learning.

b. Google cars create models of people on the road using machine learning. 
c. Amazon's "Featured Recommendations" uses machine learning together with prior browsing history.

Full descriptions of AI paradigms and constituents can be found in Artificial Intelligence books [8-15].

In our days, the ability of AI in smart machines is progressing from handling classical repetitive tasks to the capability to adaptively carrying out continuously changing tasks. In other words, AI application is evolved along three stages, namely:

i. Stage 1: Assisted Intelligence (tasks don't change, machines learn, tasks are automated).

ii. Stage 2: Augmented Intelligence (changing nature of tasks, humans inform machines, machines inform humans).

iii. Stage 3: Autonomous Intelligence (changing nature of tasks, decisions are automated, machines learn continuously).

Assisted intelligence allows automating repetitive and routine manual and cognitive tasks. Augmented intelligence helps to handle more complex situations and enhance human decision making. Finally, when machines are able to learn enough about the situation and make reliable decisions that humans can trust, they can become autonomous (autonomous intelligence).

Today there are numerous artificial tools that can be used in research and applications. These tools can be classified as:

a. AI tools for personal use.

b. AI tools for business use.

c. AI tools for industry specific business.

A list of AI tools for each of the above categories is provided in [16].

\section{Synergy of IoT and AI}

Both AI and IoT are now at very mature states and their synergy promises a lot of benefits. IoT, which by many industry thinkers is considered to be the driver of the Fourth Revolution, has inspired a variety of technological advances and changes covering a wide range of fields. Many thinkers believe that IoT really needs AI, and in fact that the future of IoT is AI [17]. They anticipate that in the near future most IoT implementations will make visible use of AI techniques and tools (particularly machine learning and reasoning algorithms and software tools). Actually, IoT and AI have been worked together in many business and other areas since quite some time. IoT collects data (actually, huge amounts of data) and AI is the proper tool to make sense of huge amounts of data. AI is the engine that performs 'analysis', processes the data, and 'makes decisions' based on this data. AI enables 'understand patterns' and therefore helps to make more informed decisions. The use of machine learning, along big data, has opened new opportunities in IoT. One can already see the synergy of these systems at a personal level in devices such as Google Home and
Amazon's Alexa [17]. Collecting data is one thing, but sorting, analyzing, and making decisions, on the basis of that data, is entirely another thing. Clearly, to be more useful in IoT, AI should develop more accurate and more rapid algorithms and tools (Figure 6).

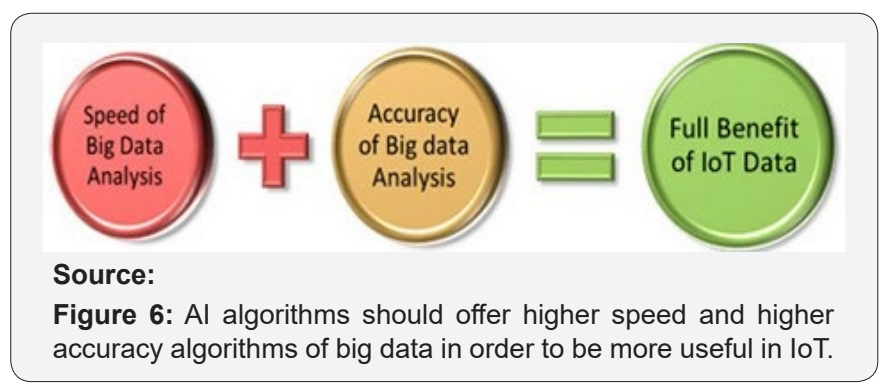

IoT supported by AI can provide the best way for enterprise stores to gain more from their store operations and assure their sustainability in the long run. Using IoT/AI retailers can, among others, minimize theft and maximize purchases through cross selling.

The operations required in $\mathrm{AI} / \mathrm{IoT}$ data analysis are the following [17]:

a. Preparation of data (define and clean pools of data).

b. Discovery of data (find useful data in the defined pools of data).

c. Visualization of streaming data (deal with streaming data on the fly, discovering and visualizing data in smart and fast ways, such as to assure rapid decision making without delay).

d. Time series accuracy of data (keep high the confidence level in collected data with high accuracy and integrity of data).

e. Predictive/advance analysis of data (make predictive decisions on the basis of collected data).

f. Real time geospatial and location [logistical data] (Marinate smooth and under control the flow of data).

A discussion on the innovation potentials and pathways merging AI, cyber-physical systems (CPS), and IoT is provided in [18], where a technology forecast is given based on extensive descriptions and developments by field, and also based on interaction traits. According to Sudha Jamthe the junction of IoT and AI constitutes the so-called 'cognitive IoT' [19]. In [20] a number of examples are provided that show how AI and IoT can work together. One of them refers to the air condition equipment of buildings and examines what happens in a very hot day in which the local utility is experiencing brownouts. In this case the system could overflow, and the utility staff would need to spend time and money to face angry customers asking for restoration of the service. If the thermostats of the buildings' equipment and the utility are connected to an IoT system, the utility staff can see how many air conditioning devices are connected to the system and react by turning everyone's 
thermostat up 3 degrees, thus preventing a brownout. A built-in AI system could do the same job automatically, whereas a more sophisticated AI system could proactively turn thermostats 3 degrees at homes and nonessential business, while at the same time keeping thermostats stable in hospitals and refrigerated warehouses.

In [21] the dynamics between AI and IoT is examined. It is argued that $\mathrm{AI} /$ Machine learning for data science is much more than applying statistical predictive algorithms to an IoT. Therefore, it is proposed that there is a need for a new type of engineer, viz. an engineer with knowledge of electronics (IoT), $\mathrm{AI}$ Machine learning, robotics, cloud, and data management. It is also argued that data science for IoT is different from traditional data science. Data science for IoT involves work with time series methods, such as autoregressive moving average methods and the like. In [22], it is explained why IoT, Big Data, and AI are three essential technologies, the synergy of which will drive the next generation of applications. It is argued that big data fueled by IoT is powerful on its own, and so is AI, but together they are the superpowers in the digital universe. Thinkers in the information field anticipate the size of the digital universe will double every two years leading to a 50 -fold growth from 2010 to 2050. For meaningful results, AI needs Big Data. Actually, AI can resolve the Big Data analytics issue.

IoT data involve the following [17]: a. Smart city data providing information that helps to predict accidents and crimes.

b. Data helping to optimize productivity across industries via preventive maintenance of devices and machines.

c. Data used in communication of automated driving vehicles.

d. Data creating truly smart homes with connected appliances.

e. Health related data giving doctors real-time insight information from biochips and pacemakers.

Humans are not able to understand and handle with standard methods large amounts of data of the above type. They need to develop new ways to analyze the performance data and information created by huge numbers of smart devices/objects. To get the full benefit of IoT data, the speed and accuracy of big data analysis should be considerably improved (Figure 2). Moreover, the continuous advances of AI cause AI to converge with IoT, to the extent that it is quickly becoming indispensable to IoT solutions. The principal elements of IoT, viz: connectivity, sensor data, and robotics, will ultimately lead to a need for almost all devices to become intelligent. In other words, IoT needs smart devices and machines. As AI convergence with IoT continues, the ongoing growth of IoT is being driven by six factors, of which the most powerful is the advent of big data and cloud/fog computing [23] (Figure 7).

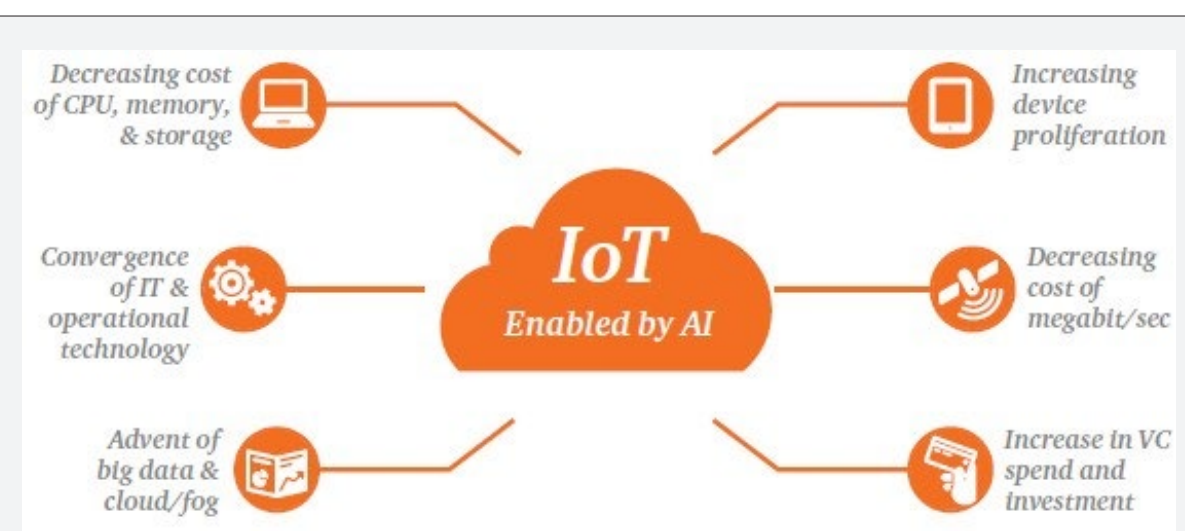

Source: [21].

Figure 7: Factors driving the growth of loT.

The challenges facing AI in IoT include:
A. Privacy/Security/Safety
B. Complexity
C. Compatibility
D. Ethical issues

The IoT applications can be classified in several ways. One of them is the following [24]:

a) Personal and home (the sensor information is collected and used by the individuals who directly own the network).

b) Enterprise (this category includes IoT within work environments, namely offices, companies, organizations, etc.)

c) Utilities (this category includes systems that offer service optimization, such as water network monitoring, smart and grid metering).

d) Mobile (this category includes urban traffic, smart transportation, smart urban traffic, smart logistics, etc.). 
Another classification of IoT application domains is the following, the contents of which are shown in Figure 8:

i. Home and building

ii. Transportation

iii. Health

\section{iv. Logistics}

v. Precision agriculture

vi. Smart industry

vii. Smart retail

viii. Smart environment

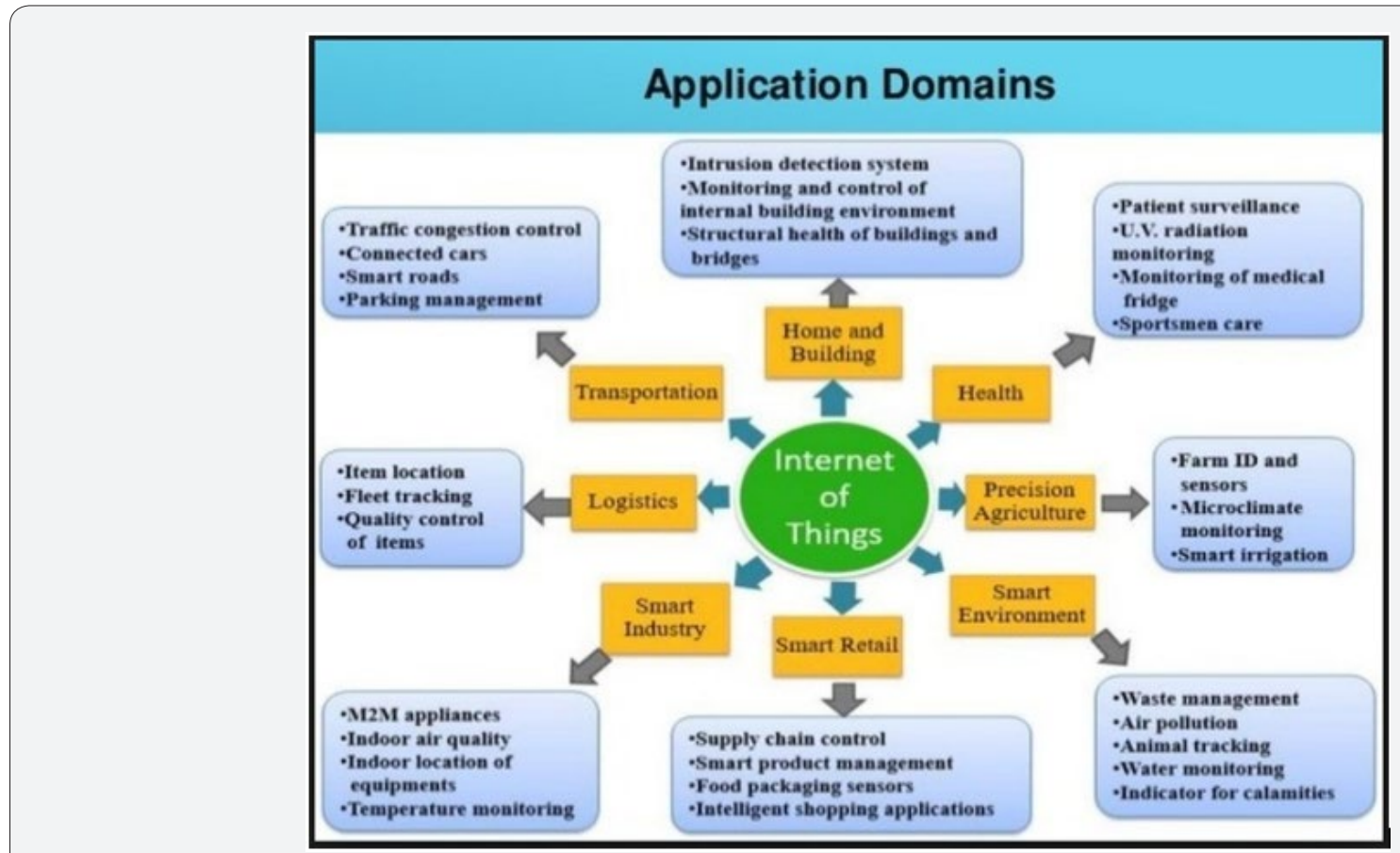

Source: [21]. https://www.quora.com /What-is-IOT-internet-of-things-What-role-does-it-play-in-our-day-to-day-life Figure 8: loT application domains.

IoT-AI provides many benefits. For example a smart hotel using AI-based IoT provides to its customers the following:

a. Smart booking system.

b. Flexibility in room temperature control.

c. Helpful information selection based on customers.

d. Customer history re-synchronization by returning guests.

e. Real-time support to customers on online platform to face their problems.

The benefits of AI-IoT in retail operations are discussed in [25], where the applications of IoT to retailers are outlined, and a number of major companies that offer data-driven personalization and customer service adopting AI and IoT are listed. In summary, the benefits of AI-IoT applications in brickand-mortal store environments include the following [25]:

1. IoT makes operations more efficient. This is achieved because of the ability of connected devices to track inventory levels in real time.

2. IoT helps retailers to improve the customer store journey by increasing engagement via devices such as smart mirrors.

3. IoT improves efficiency in retailer/supplier relationship. This is facilitated by the partnership of retailers with suppliers who are able to respond promptly and efficiently to frequent orders driven by the retailer's real-time inventory tracking system.

4. AI enables retailers to provide a personalized and straightforward shopping experience and scale up the use of customer data. This includes customization of shopping recommendations, and e-commerce and m-commerce portals layout and promotion.

5. AI helps retailers to drive the sales and forecast demand. With AI, retailers can maximize the probability of having the right goods in stock, which assures faster fulfillments and leaner inventory operations.

6. AI helps retailers to analyze customer data so as to get a better understanding of customer/consumer behavior, in order to adapt the approach through which the enterprise interacts with shoppers and predicts consumer demand. 
7. AI enables retailers to operate 'chatbots' that imitate the customer's interaction with a customer care or sales associate, in order to understand what the best way is of responding to the customer's need

8. AI enables computers to observe, exploit and strategize data, and implement strategy.

Figure 9 shows the three basic stages of forecasting customer demand using AI [25]. From the above it follows that to get maximum benefit from IoT-AI, retailers should do the following:

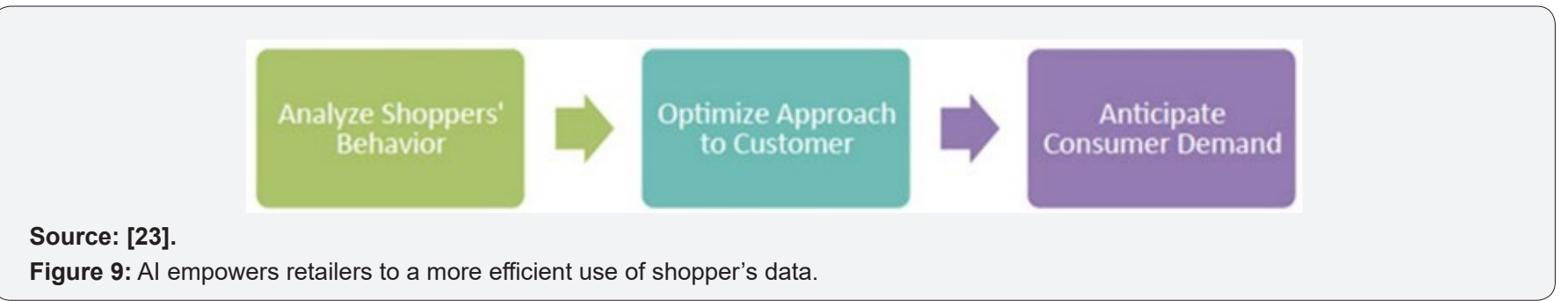

A. Act quickly to adapt to competitors that adopt new technologies.

B. Handle data with care and set-up proper strategies for handling consumer data.

C. Review relationship with suppliers and redefine the relationship with other supply-chain players.

Big retailers that run IoT/AI-based systems include: Amazon Go, Walmart, Carefour, Catalyst, Smartrac, Rebecca Minkoff Connected Store, Panasonic, and Coresight Research, 2018. The ten top industries that adopted AI-IoT are:
a. Smart manufacturing
b. Smart retail
c. Smart automobile
d. Smart health
e. Smart transportation
f. Smart education
g. Smart finance
h. Smart entertainment
i. Smart home
j. Smart security/surveillance

\section{Industrial Internet of Things}

The incorporation of robotic issues into the wider IoT was called by ABI Research "Internet of Robotic Things" (IoRT). IoRT is actually concerned with machine to machine (M2M) communication between robots and devices in an ecosystem where data are employed to drive insights and actionable outcomes. The robot is an intelligent device in the sense that it can monitor events and fuse data from several sources in order to determine and execute a best course of action, e.g., a move through the physical environment and manipulation of objects in this environment in a desired way. Potential applications of IoRT include: a. Use a robotic device to check if a car is allowed to use a given park lot in a corporate parking area.

b. Collaboration of IoRT and humans in a manufacturing unit to make operational and other decisions.

c. Use the concept of IoRT to add more flexibility and adaptability to intelligent transportation systems (ITS).

d. Use of IoRT for elderly assistance and domestic cleaning.

One of the major application areas of IoT is the socalled "smart industrial automation". With the aid of IoT infrastructure, advanced sensor networks, wireless connectivity, and M2M communication, conventional industrial automation is modernized completely. Most industries (small and large) have already adopted and are using IoT enhancements. IoT based industrial automation represents the present state of automation, called "industrial automation 4.0" or "Industrial Automation Internet of Things" (IAIoT). An umbrella term that covers both the IoRT and IAIoT is the term "Industrial Internet of Things" (IIoT). IIoT also embraces industrial control systems and manufacturing systems. IIoT involves smart connected assets (machines, engines, robots, actuators, power grids, sensor clouds, etc.) that operate as part of a larger system or system of systems that comprise the smart manufacturing system. The connected assets can monitor, collect, analyze, exchange, and instantly act on data/ information to automatically and intelligently change their performance or their environment. An analysis framework for IIoT devices is provided in [26], which gives a practical classification scheme with reference to IIoT security aspects. IIoT offers reduced cost structure, and increased operational efficiency, accompanied by higher quality of products (fewer failures, more efficient materials' sourcing, etc.).

A pictorial illustration of the components that are included in industry 4.0 and create the so-called "smart factory" is given in Figure 10. The dominant components are: IoT/IoRT, cyberphysical systems, and cloud computing. A good interface that can be an assistant to the engineers is the chatbot which is easy 


\section{Robotics \& Automation Engineering Journal}

to use, provides real-time interaction with IoT and robots, has a question-answer structure, and is a perfect interface for AI. A

typical IoRT-based robotic manufacturing shop floor is shown in Figure 11 [27].

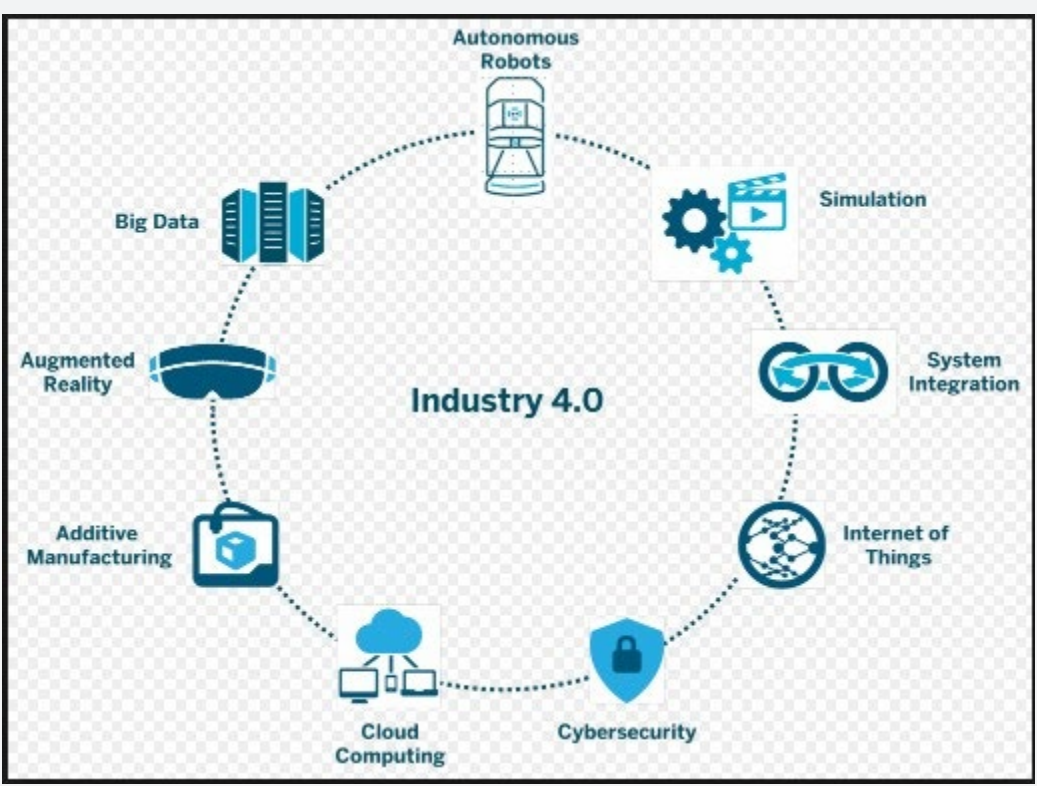

Source: https://chatbotslife.com/the-role-of-chatbot-in-industry-4.0-acf2b0ac8bb6

Figure 10: Constituent components of "industy 4.0". A novel interface for the communication of humans, sensors, robots, and big data is a "chatbot".

Source: [27].

Figure 11: A smart loRT-aided manufacturing line.

A discussion of the challenges and technical solutions concerning the IoT for industrial automation is provided in [26], including the identification of challenges for long-living IoT -aided industrial automation with enormous complexity. Some of the IIoT challenges considered in [28] are the following:

a. Latency and scalability of data (this issue can be faced through localization of computation).

b. Mixed criticality (this challenge can be managed through system partitioning). c. Scalable and secure real-time collaboration (this can be achieved through the so-called 'zero-configuration networking' method).

d. Fault tolerance (this issue can be managed through networking redundancy or local fault detection near the end devices).

e. Functional safety (This can be addressed by separating the safety related issues from IoT). 


\section{Case Studies}

In the following, a number of case study and application examples will be listed which give a good picture of the range of IoT/IIoT applications, especially in automation and robotics.

\section{Home automation}

A IoT-based monitoring and control system for home automation is described in [29]. This is an embedded system that uses a PIC microcontroller which provides intelligent energy preservation. It can control and automate most of the home appliances (such as lights and fan on/off) through a manageable smart phone-based android interface. The components are connected to the embedded micro-web server through LAN or WiFi module for accessing, monitoring, and controlling devices and appliances using android-based smart phone applications. The system also keeps track of status of the devices.

\section{Oil field production}

An oil and gas company use IoT to optimize oilfield production. To this end the company is using sensors to measure oil extraction rates, temperature, well pressure, and other variables for 21,000 wells. The frequency of readings is $90 \times$ day $\times$ variable. The number of data collected is about 18,900 per day. To convert raw IoT data into business data and tangible benefits, the company employs analytics to realize both direct and opportunity cost associated with the analysis of IoT data. The synergy of IoT industrial analytics resulted in persistent significant advancements [30]. Two other AI-IoT case studies presented in [30] are the following:

a. A smart municipality water metering system covering all residential and commercial water meters.

B. Water meters were mounted on 66,000 devices that used to be manually read and recorded.

a. An international truck manufacturer outfitted more than 100,000 trucks with sensors for predictive maintenance. The system is scheduling repairs automatically when needed and orders the required parts for each repair. More than 10,000 data points are transmitted per day for each track.

\section{ABB Smart robotics}

This multinational power and robotics Company adopted IIoT for developing an efficient predictive maintenance system. A large number of connected sensors monitor the maintenance requirements of its robots (across five continents) and trigger repair before parts break. The Company's collaborative robotics is also based on IoT. Its YuMi model can collaborate with humans through Ethernet and industrial protocols (Profibus, Device Net, etc.) [31].

\section{Boeing smart manufacturing}

This multinational aviation Company has strongly deployed IIoT technology to drive efficiency in all of its factories and supply chains and is continually increasing the number of sensors embedded in its planes. Currently, Boeing is working towards making service offerings very important, while being at the top of information providers in aviation [31].

\section{KUKA connected robotics}

This Company has an IoT policy which extends to entire factories. For instance, as mentioned in [31], Jeep asked KUKA to help build a factory that could produce a car body every 77 seconds. KUKA responded by helping the Company to build an IoT-based factory with hundreds of robots connected to a private cloud. In this way more than 800 vehicles can be produced each day.

\section{Fanuc: Smart factory down time minimization}

Fanuc, a robot maker has put much effort to reduce down time in industrial facilities. The Company uses sensors within its robotics in tandem with cloud-based analytics. In this way the Company is able to predict when failure of a component such as a robotic system or process equipment is about to occur. The outcome of this effort is the so-called "Fanuc's Zero Dynamics System" [31]. In [30] a total of 30 top real IIoT applications are described. Three of them, besides those described above, are the following:
a. Magna Steyr: Smart automotive manufacturing.
b. Kamatsu: Innovation in smart mining and heavy equipment.

c. Shell: Smart oil field Innovator.

\section{IoT-aided robotic applications}

The range of applications of IoT -aided robotic systems is very wide, and includes robots used in the manufacturing /automobile industry, health care, military, deep underwater exploration, space exploration, rescue, and security operations. IIoT helps to solve a large variety of industrial problems from temperature/pressure monitoring, to power consumption monitoring, to electrical grid monitoring, and so on. IoT applications include detection of perimeter intrusions in airports, railway stations, and ship ports. IoT paired with AI (perception, natural language understanding) enables efficient human robot interaction. Cloud robotics play a key role in enabling robot functions, e.g., mobility, sensing, manipulation, etc. IoT-based robotic systems also find application in short range communication technology, protocol design, and security assurance in smart pervasive environments. An example of cloud robots is a driverless (autonomous) car which is connected to the internet to get access into the database of maps and satellite imagery. Using sensor fusion to exploit streaming data from its camera, and the global positioning system (GPS), together with 3D sensors, a driverless car can localize its position accurately (within centimeters). Figure 12 shows the capabilities of a driverless car that are achieved through proper sensors. This car is also connected to an IoT platform. 


\section{Robotics \& Automation Engineering Journal}

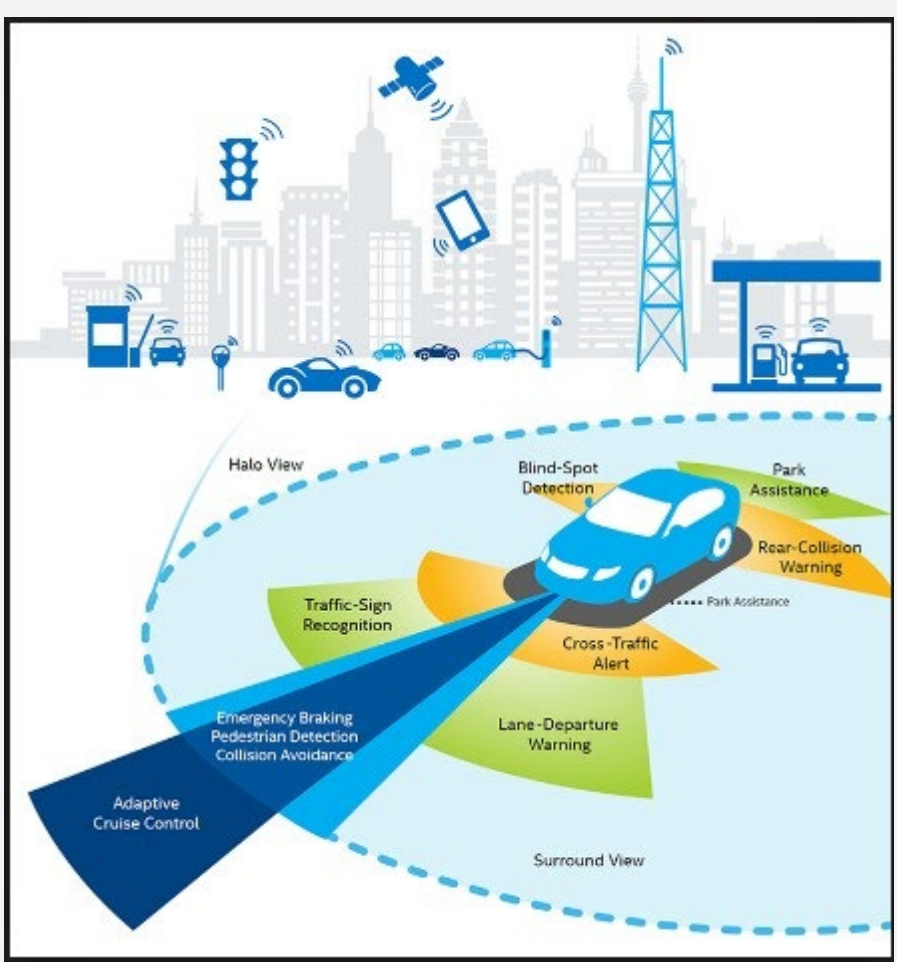

Source: https://blogs.intel.com/iot/2015/11/23/building-the-next-generation-car-with-intel-iot

Figure 12: Driverless car connected to the Internet of Things for $360^{\circ}$ awareness. The car is equipped with sensors for safe movement in the traffic.

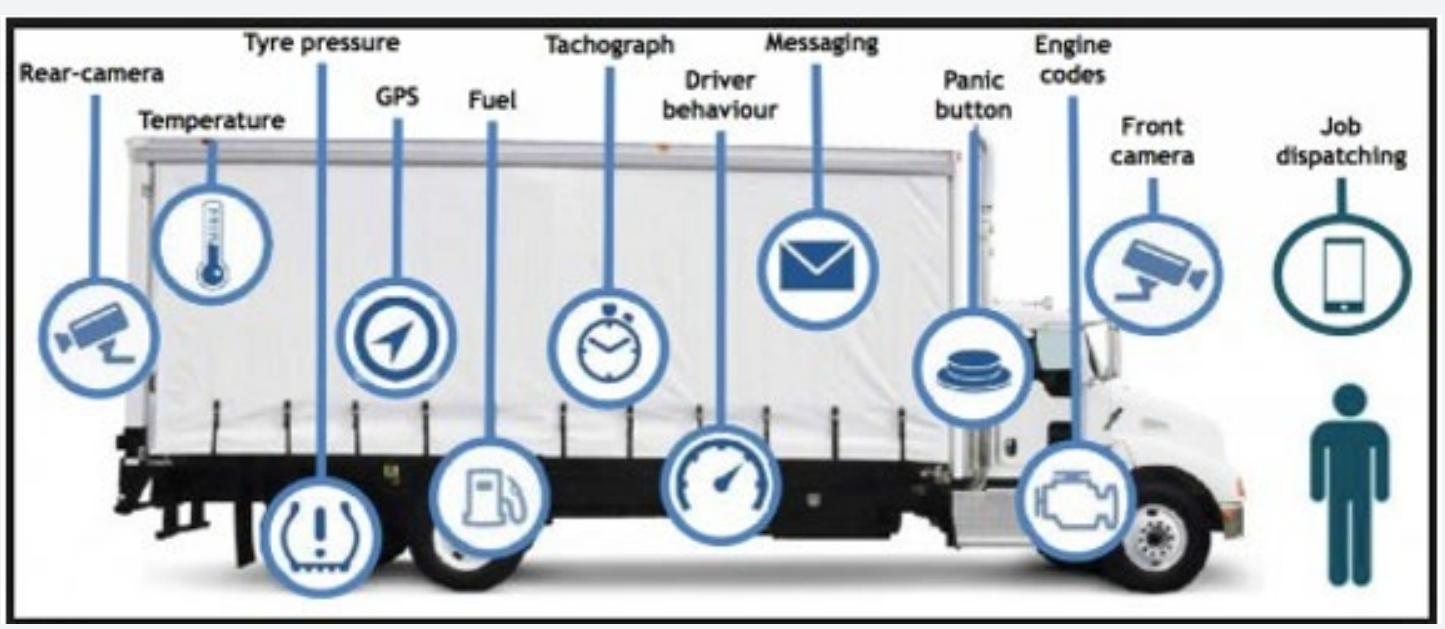

Source: https://rctom.hbs.org/submission/coca-cola-original-formula-in-an-iot-world

Figure 13: loT connected truck.

Figure 13 depicts a typical IoT/AI-aided truck with its sensors. The transportation benefits obtained if the vehicles are connected to the IoT, and travel in smart roadways, are the following:

a. Transportation efficiency (real-time traffic is secured, transit and parking data for maximum efficiency and minimum congestion are generated).

b. Low operating costs (preventive maintenance driven by operating data and diagnostics improves warranty and services). c. Improved safety (connected vehicles 'talking to each other' enable cooperation and assure crash avoidance and safety).

Of course, it should be noticed that, as is always the case with IoT-aided applications, any part of a vehicle that talks to the outside world is vulnerable to potential cyber-attack and special measures should be taken. An important application of IoT/AI-aided robots is the home security. An example of these robots is the "AppBot" home security toy robot, a WiFi controlled robot throughout the Internet (Figure 14). 


\section{Robotics \& Automation Engineering Journal}

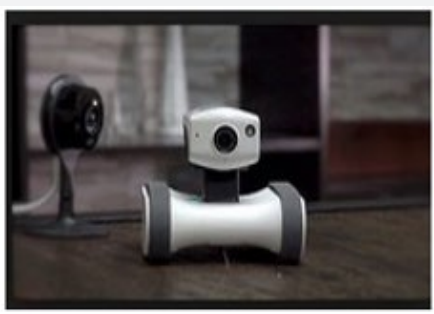

(a)

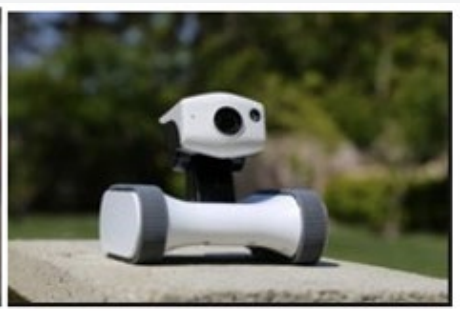

(b)

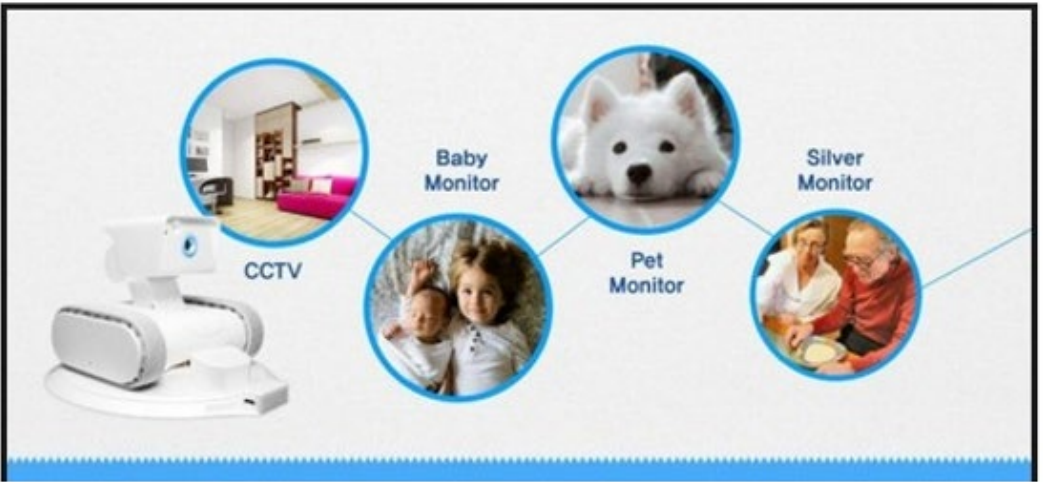

(c)

Source: https://iot.do/devices/appbot-security-robot

Figure 14: The AppBot smart home security toy robot. (a) The robot indoor, (b) The robot outdoor, (c) Uses of the robot (baby monitor, pet monitor, silver monitor).

The robot provides the following:

a. Live view and remote control.

b. Snapshot and video recording.

c. Motion detection and tracking while communicating with a human. d. Clear two-way talk.

e. It can be connected with the router of the house and provide access from everywhere in the world.

f. If intruders appear in a house the robot can spontaneously rotate itself to capture them in seconds and send alarm notification messages.

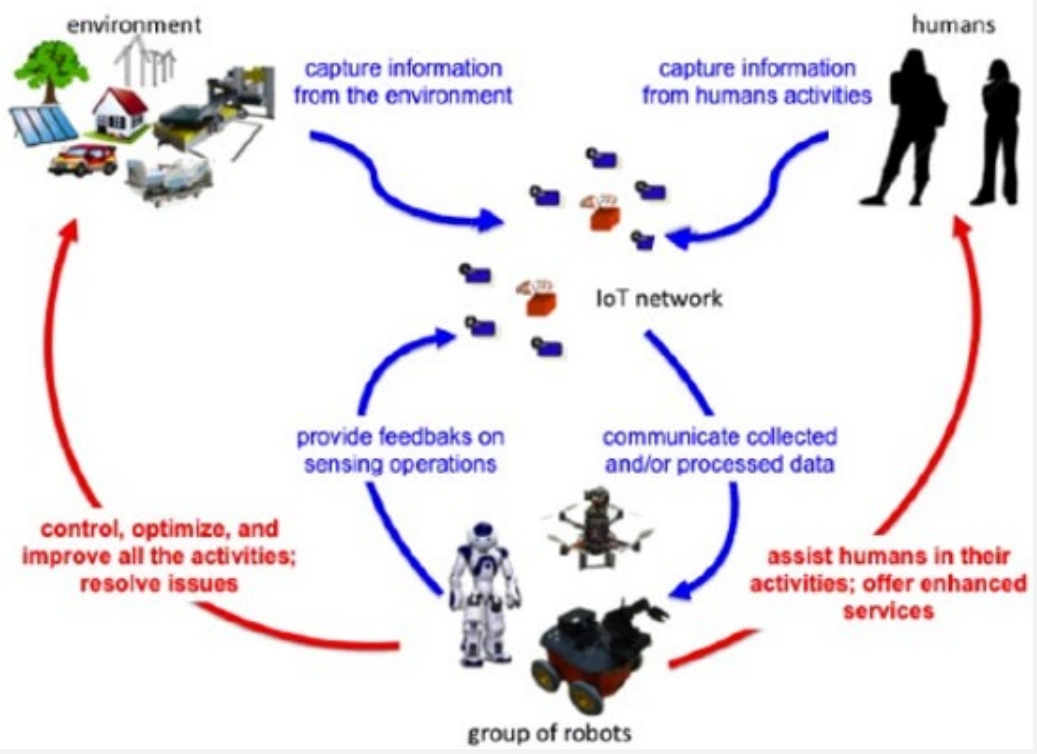

Figure 15: Global scenario for loT-aided robotic applications [38]. 


\section{Robotics \& Automation Engineering Journal}

A general comprehensive discussion of IoT-aided robotics applications and implications is presented in [32]. This paper includes state of art issues, highlights the most important challenges, describes currently available tools, and explains why a joint investigation of IoT-aided robotics problems is needed by research teams with complementary skills. Figure 15 shows an IoT-AI-aided robotics scenario created in [32]. The IoT-aided robotic applications discussed in [32] are the following: healthcare, industrial plants and smart areas, military operations, and rescue operations.

IOT-Aided Industrial Automation

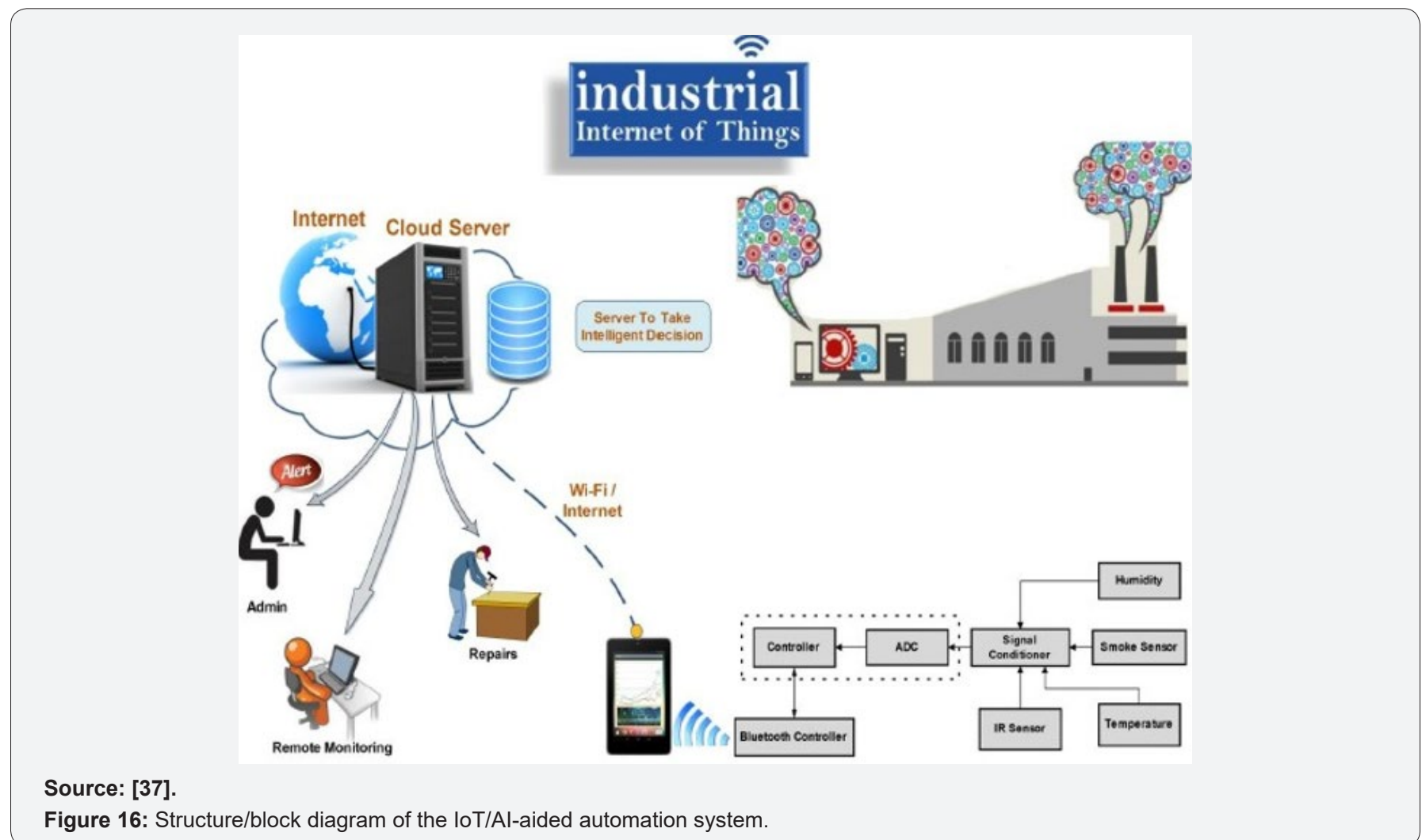

Here, a representative system that generates alarms/ alerts, and makes intelligent decisions in IoT/AI-aided industrial automation systems is outlined [33]. IIoT enables remote sensing and control of objects across available network infrastructure. The structure of this system is as shown in Figure 16.

The system is equipped with sensors (temperature, pressure, humidity, vibration, intrusion, etc.) to percept the environment and the objects' conditions. The analog signals are inputted to the android device which checks the thresholds set by the system administrator and compare them with incoming analog signals. When an uneven/anomalous condition is encountered, special devices (e.g., Buzzer, Alarm, fan, etc.) are employed to take proper measures such as sending an Alarm/ Alert to the system administrator. Then, with the aid of AI the system takes appropriate adequate steps for resolving the problems on the basis of past experience and similar conditions stored in the data base. The cloud is appropriate for use as database of scalability. Cloud computing in industrial IoT provides computing services like storage, servers, networking, software, database, analytics, etc. The cloud-based storage allows the remote database to save data files rather than keeping files on a local storage device. With cloud computing the sharing on networks is much faster than access via other networking. Figure 17 gives a pictorial illustration of cloud computing use in the manufacturing sector.

\section{Concluding Remarks}

The Internet of Things has already been established as a major field of multidisciplinary nature that promises to offer services to society of enormous value. Particularly, its recent integration with AI has already exhibited a great success in complex and large-scale real-life applications. The field has reached a very mature state, but scientists and engineers predict that a much more advancement will take place in future with unimaginable beneficial implications for the human life. In this article we have attempted to compile a holistic overview of the IoT field and its synergetic integration with AI in robotic and industrial automation applications. In industrial/robotic automation IoT enables successful facility management, production flow monitoring, inventory control, logistics, supply chain management, and robotic operation. Although IoT security has received from the beginning of the field considerable attention, the solutions derived and used so far were not proved completely successful. Actually, security 


\section{Robotics \& Automation Engineering Journal}

and privacy still remain the biggest challenges in IoT and IIoT applications. Another problem which is still largely open is the problem of designing distributed and many-to-many IoT/IIoT. This will require the development of new kinds of interconnectedness, interrelationship, and interdependence, such that IoT/IIoT will offer a collective and collaborative resource in which individuals can contribute at their wish. A further topic of high value in the implementation and application of IoT/IIoT-AI is the study of ethics/morality which determines the principles and rules that have to be applied in the field for securing an ethical/moral use of IoT/AI in everyday life applications [34-40].

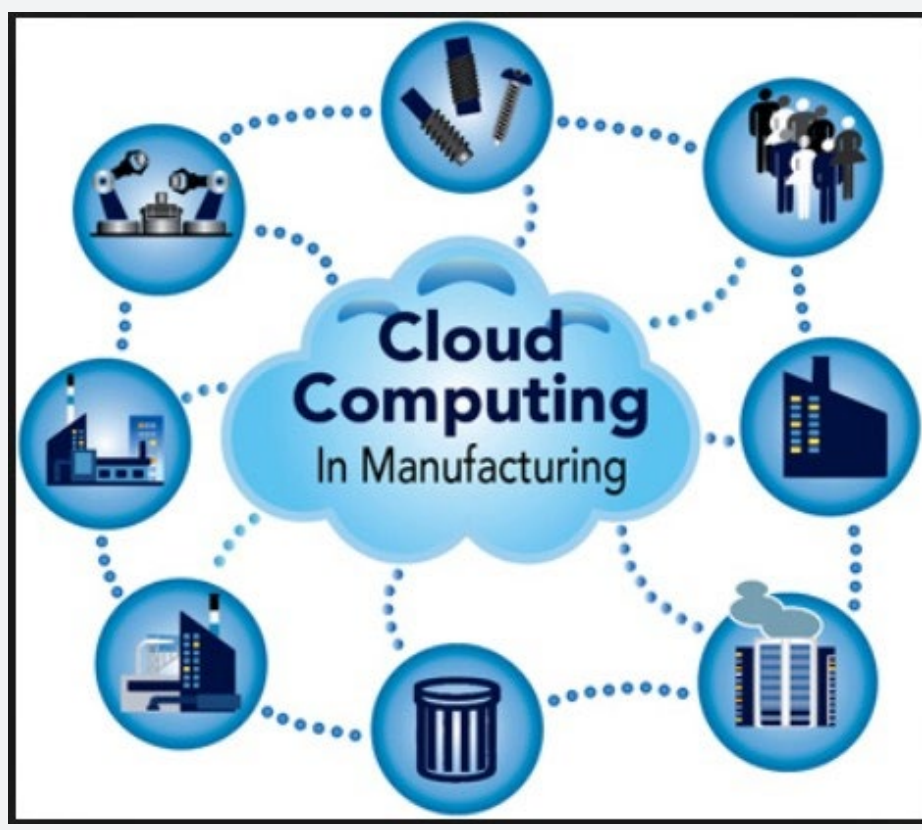

Source: www.evidentsoftware.com/latest-cloud-solutions-for-it-industry

Figure 17: Cloud computing in manufacturing sector.

\section{References}

1. Patel KK, Patel SM (2016) Internet of Things-IoT: Definition, characteristics, architecture, enabling technologies, applications, and future challenges. International J of Engineering Science and Computing 6(5): 6122-6131.

2. Atzori L, Iera A, Morabito G (2010) The internet of things: A survey. Computer Networks 54(15): 2787-2805.

3. Sarma S, Brock D, Aston K (2000) The networked physical world. Proposals for engineering the next generation of computing, commerce and automatic identification. White Paper of the MIT Auto-ID Center, Cambridge, Massachusetts, USA.

4. Ashton K (2009) That 'Internet of Things' thing.

5. ITU: The Internet Executive Summary (2005) ITU Internet Reports.

6. http://iot.ieee.org

7. van den Hoven J (2012) Fact sheet-Ethics Subgroup IoT-Version 4.0. IoT Expert Group, Delft University of Technology. The Netherlands.

8. McCarthy J (2007) What is Artificial Intelligence?

9. Feigenbaum EA (1983) The Fifth Generation.

10. Barr A, Feigenbaum EA (1971) Handbook of Artificial Intelligence. Pitman, London, England.

11. Rich E (1984) Artificial Intelligence. Mc Graw-Hill, New York, USA.

12. Popovic D, Bhatkart VP (1994) Methods and Tools for Applied Artificial Intelligence. Marcel Dekker, Inc, New York, Basel, USA.

13. Forsyth R (2017) Expert Systems. Chapman and Hall, Boca Raton, Florida, USA.
14. Bowerman R, Glover P (1988) Putting Expert Systems into Practice.Van Nostrand Reinhold, New York, USA.

15. Ericsson (2018) Artificial intelligence and machine learning in the next generation systems.

16. Hänel L (2017) Medium Corporation.

17. Banafa A Internet of Things (2007) Why IoT needs AI.

18. Klumpp M (2018) Innovation potentials and pathways merging AI, CPS, and IoT, Applied System Innovation 1(1): 5.

19. Jamthe S (2016) Business Junction of IoT and AI.

20. Sappin DE (2017) How AI and IoT must work together: R2 uses big data to develop IoT solutions for enterprise customers.html

21. Jaokar A (2017) The dynamics between AI and IoT.

22. Misauer I (2017) Io T, big data and AI: The new superpowers in the digital Universe.

23. (2015) Leveraging the upcoming disruption from AI and IoT: How artificial intelligence will enable full promise of the internet of things.

24. Cubbi J, Buyya R, Marasic S, Planiswami M (2013) Internet of Things (IoT): A Vision, Architectural Elements, and Future Directions. Future Generation Computer Systems 29: 1645-1660.

25. (2018) Deep Dive: The future customer experience-AI and IoT in retail.

26. Boyes H, Hallaq J, Cunningham, Watson T (2018) The industrial internet of things (IIoT): An analysis framework. Computers in Industry 101: $1-12$.

27. Alba M (2017) Thing Worx and Deloitte release plans for industry specific IoT solutions, Engineering Com. 


\section{Robotics \& Automation Engineering Journal}

28. Breivord HP, Sandström K (2015) Internet of Things for industrial automation: Challenges and technical solutions. Proceedings of IEEE Conference on Data Science and Data Intensive Systems (DSDIS) pp 532-533.

29. Gurav U, Patil G (2016) IoT based interactive controlling and monitoring system for home automation, International J of Advanced Research in Computer Engineering and Technology (IJARCET) 5(9): 2392-2396.

30. Bolen A (2018) 3 IoT examples from 3 industries: Real world implementation achieving results today.

31. Buntz (2017) Industrial IoT: The top 20 Industrial IoT applications.

32. Grieco KA, Rizzo A, Colucci S, Sicarri S, Piro G, et al. (2014) IoT-aided robotics applications: Technological implications, target domains, and open issue. Computer Communications 54(1): 32-47.

33. Deshpande A, Pitale P, Sanap S (2016) Industrial automation using Internet of Things. International Journal of Advanced Research in Computer Engineering and Technology, (IJARCET) 5(2): 266-269.

34. Tzafestas SG (2016) Roboethics: A Navigating Overview. Springer, Berlin, Germany.

This work is licensed under Creative

Commons Attribution 4.0 License

DOI: 10.19080/RAEJ.2018.03.555621
35. Tzafestas SG (2018) Information, Communication, and Automation Technology Ethics in the Knowledge Society Age, Hauppauge. Nova Science Publishers, New York, USA.

36. Chowdhury AR (2017) IoT and robotics: A synergy.

37. Dalataela MP, Accentura N, Griego L, Boggia G, Dohler M, et al. (2013) On optimal scheduling in duty-cycled IoT using IEEE $802.15 .4 \mathrm{c}$ TSCH. IEEE Sensor J 13(10): 3655-3666.

38. Potter C, Haneke G, Silva B (2013) Machine to machine possible applications in industrial networks. Proceedings of IEEE International Conference on Industrial Technology (ICIT) 2013: 1321-1326.

39. Kuffner JJ (2010) Cloud-enabled robots. IEEE RAS International Conference on Humanoid Robots, Nashville, Tennessee, USA

40. Manasseh E (2018) Combined artificial intelligence and IoT for smart sustainable cities, $1^{\text {st }}$ Forum on Artificial Intelligence and the Internet of Things in Smart Sustainable Cities in Latin America, Buenos Aires, Argentina.

\section{Your next submission with Juniper Publishers} will reach you the below assets

- Quality Editorial service

- Swift Peer Review

- Reprints availability

- E-prints Service

- Manuscript Podcast for convenient understanding

- Global attainment for your research

- Manuscript accessibility in different formats

( Pdf, E-pub, Full Text, Audio)

- Unceasing customer service

Track the below URL for one-step submission https://juniperpublishers.com/online-submission.php 